\title{
Entrelacs
}

Cinéma et audiovisuel

14 | 2018

Marchés du film : évolutions, mutations et perspectives

\section{Festival et marché : le rôle du festival Cinélatino de Toulouse dans l'industrie du « cinéma d'auteur»}

Amanda Rueda

\section{OpenEdition}

\section{Journals}

Édition électronique

URL : http://journals.openedition.org/entrelacs/4265

DOI : 10.4000 /entrelacs. 4265

ISSN : 2261-5482

Éditeur

Éditions Téraèdre

Référence électronique

Amanda Rueda, «Festival et marché : le rôle du festival Cinélatino de Toulouse dans l'industrie du « cinéma d'auteur » », Entrelacs [En ligne], 14 | 2018, mis en ligne le 15 décembre 2018, consulté le 20 avril 2019. URL : http://journals.openedition.org/entrelacs/4265 ; DOI : 10.4000/entrelacs.4265

Ce document a été généré automatiquement le 20 avril 2019.

Tous droits réservés 


\title{
Festival et marché : le rôle du festival Cinélatino de Toulouse dans l'industrie du « cinéma d'auteur »
}

\author{
Amanda Rueda
}

1 Les trois dernières décennies ont été marquées par l'évolution des festivals vers un compagnonnage qui va bien au-delà de la programmation et de la diffusion des films. L'élargissement du répertoire d'actions festivalières s'est manifesté à travers l'organisation d'ateliers de formation et la création d'espaces formalisés de négociation où se rencontre le tandem producteur-réalisateur et les différents acteurs du marché (producteurs, distributeurs, vendeurs, programmateurs d'autres festivals). Le Hubert Bals Fund du Festival de Rotterdam, créé en 1988 et destiné à soutenir les secteurs de la production, de la post-production et de la distribution de projets cinématographiques issus de régions spécifiques (Asie, Moyen-Orient, Europe de l'Est, Afrique et Amérique latine) en est le premier exemple. Chaque festival d'une certaine envergure compte désormais son fonds de soutien ou, à défaut, une plateforme professionnelle de soutien à la production. Ces dernières prolongent les fonds institutionnels ou intergouvernementaux abondés par les gouvernements ${ }^{1}$, lesquels répondent à des politiques publiques de soutien aux industries culturelles et de promotion de la diversité. Mais elles s'en distinguent du fait qu'elles s'imposent désormais comme des rendez-vous obligés pour les professionnels, notamment français, intéressés par ces cinémas, ce qui entraine de profondes mutations dans les pratiques de coopération et de coproduction cinématographique avec les pays d'Amérique latine et plus largement les pays dits du Sud. Le glissement est manifeste: d'une politique promue par les États, français en l'occurrence, à la mise en place de plateformes professionnelles à l'initiative des acteurs du secteur, et plus particulièrement des festivals.

2 À l'instar de ce que nous avons entrepris précédemment avec les festivals de cinéma ${ }^{2}$, il s'agit dans cet article d'explorer la plateforme professionnelle Cinéma en Construction, du festival Cinélatino Rencontres de Toulouse en tant qu'espace de médiation au sein du marché du film d'auteur latino-américain. À travers l'analyse des documents et des 
entretiens avec des membres de l'organisation et des professionnels latino-américains qui y participent, nous tenterons d'éclairer les enjeux de ce rendez-vous pour les cinématographies latino-américaines ainsi que les pratiques de circulation des films et des projets qui contribuent à la construction de ce que d'aucuns n'hésitent pas à qualifier d'« industrie internationale du cinéma d'auteur ${ }^{3}$ ».

\section{Festival et médiations cinématographiques}

3 À l'image du festival de cinéma, la plateforme professionnelle se structure comme un «fait de communication ${ }^{4}$ » en ce qu'elle implique des auteurs cinéastes et des communautés de réception et d'interprétation composées de professionnels du secteur. Du point de vue de la médiation, elle doit être vue comme un espace à l'intérieur duquel se tissent des relations objectives entre acteurs et donc comme instance constitutive du champ - espace de rencontre, de négociation et de positionnement mis en regard des enjeux artistiques et économiques dont sont porteurs ces cinémas -. La médiation doit être comprise ici comme la construction de sens par différents acteurs, dans une mise en relation. S'il s'agit d'acteurs qui participent à la production de films, il est alors vrai, comme l'affirme Pierre Delcambre, que « le terme de médiation ne vaudrait dès lors qu'au pluriel : de multiples "acteurs" opèrent d'incessantes médiations, et précisément les réseaux structureraient ce jeu en mettant en contact des individus partageant suffisamment de valeurs, d'intérêts... ${ }^{5}$ \%.

4 À travers leurs plateformes professionnelles, les festivals de cinéma spécialisés dépassent le seul secteur de la diffusion et l'enjeu habituel de visibilité des films pour gagner la production et la distribution cinématographiques. À ce titre, la présence des vendeurs n'a rien d'anecdotique. Le processus de médiation à l'œuvre agit ici dans le sens de l'accompagnement des jeunes cinéastes et du soutien aux professionnels engagés au sein de ce marché. Il s'agit certes d'un marché "mineur» comparé aux marchés internationaux associés à des grands festivals comme Cannes, moins soumis aux impératifs économiques que sont la concurrence professionnelle, la concentration, les pratiques de marketing et la rhétorique du box-office. À ce marché participe un éventail de sociétés de production et de distribution moyennes et petites, "une variété qui implique aussi, pour les films en question, des carrières sensiblement différentes selon le type de société ${ }^{\star} »$. La fidélité et l'élargissement du réseau des professionnels et des institutions soutenant le dispositif (plus de 200 en 2017) montrent aussi bien la place qu'il occupe désormais dans l'internationalisation des films et des cinématographies concernées que son caractère de niche vis-à-vis des grands marchés (10000 accréditations au Marché International du Film du festival de Cannes depuis le milieu des années $2000^{7}$ ). En peu de temps, le dispositif Cinéma en Construction du Festival Cinélatino Rencontres de Toulouse s'est positionné dans cette niche des "indépendants ", se définissant comme un des lieux de référence du circuit européen-latino-américain.

\section{Cinéma en construction. Une plateforme professionnelle pour un festival spécialisé}

5 L'origine de Cinéma en Construction montre combien la création des plateformes professionnelles se situe au-delà d'une stratégie de marque des festivals visant à accroître 
leur notoriété 8 . Certes, l'essor de ce dispositif n'est pas anodin dans le retentissement de Cinélatino au sein du circuit festivalier international et auprès de la critique française et des professionnels européens et latino-américains, mais revenons d'abord à sa genèse.

Après avoir épuisé toutes les pistes de financement pour la postproduction, deux jeunes Argentins, Matias Mosteirin, producteur, et Israel Adrian Caetano, réalisateur, apportent en mars 1999 une cassette VHS avec un montage inachevé de leur film Bolivia et demandent aux organisateurs du festival Cinélatino Rencontres de Toulouse de les mettre en contact avec les professionnels français présents. Bolivia est ainsi le premier film projeté sous une forme inaboutie dans une petite salle de la Cinémathèque de Toulouse ${ }^{9}$. Ce sont ensuite les liens entre Manuel Pérez Estremera et José María Riba, anciens amis et collaborateurs des Rencontres de Toulouse, avec le Festival International de San Sebastián - l'un président et l'autre membre de la direction et par ailleurs délégué de la Quinzaine des Réalisateurs - qui favoriseront le rapprochement entre les deux festivals à partir de 2002 avec la transformation des Working progress organisés pendant deux éditions à Toulouse en rendez-vous régulier baptisé Cinéma en Construction. Né à une période de mutations cinématographiques dans plusieurs pays du continent, le dispositif dynamise la production et la circulation de ces films à travers la mise en place d'un espace où des films inachevés auront la possibilité d'être projetés auprès d'un public de professionnels afin de trouver un financement pour la post-production.

7 Dès lors, un appel à concours est lancé deux fois par an, une édition se déroulant à Toulouse au mois de mars et une autre à San Sebastián au mois de septembre. Les deux festivals jettent désormais une nouvelle passerelle entre les cinéastes d'Amérique latine et les professionnels européens en leur proposant la découverte, en avant-première, des premiers montages. La dimension européenne offre en outre l'occasion d'élargir le champ des partenaires à des professionnels réunissant les différentes filières de la chaîne production/distribution de deux pays, l'Espagne et la France, ce qui donne aux films sélectionnés davantage de chances d'aboutir.

8 Pendant deux jours, les professionnels sont réunis dans une salle autour des six films de la sélection, ponctués par la présentation des besoins du film par l'équipe (réalisateur et producteur) et par sa projection, suivie d'un échange avec le public. À la fin de la seconde journée, trois prix sont décernés : le Prix de Cinéma en Construction Toulouse, lequel assure au projet lauréat son achèvement ${ }^{10}$, le Prix des Distributeurs et des Exploitants Européens décerné par Europa Distribution et la Confédération Internationale des Cinémas d'Art et d'Essai (CICAE) qui s'engagent à promouvoir le film primé dans un réseau de 130 distributeurs indépendants, et le Prix Spécial Ciné + En Construction, qui garantit l'achat du film par la chaîne Ciné + pour un montant de $15000 €$ et sa diffusion à l'antenne.

9 Sur 200 films visionnés en moyenne chaque année, douze sont sélectionnés (six à San Sebastián et six à Toulouse) par une commission composée de dix personnes (cinq membres des équipes de chacun des festivals). En 2018, cette commission a visionné 201 films représentant 16 pays. Au cours de ses 33 éditions, Cinéma en Construction a sélectionné 189 films et attribué 31 Prix Cinéma en Construction de l'Industrie. En l'occurrence, l'analyse quantitative des films visionnés entre 2011 et 2017 permet d'esquisser une radiographie des cinématographies nationales. Sur un total de 652 films, l'Argentine s'avère effectivement l'industrie la plus solide avec 192 films à son actif (29,45\%), suivie du Mexique (105 films), puis du Chili (77) et du Brésil (73), et enfin de la Colombie (51). Les autres pays ne comptent qu'entre 2 et 22 films (Équateur, Guatemala, 
Porto Rico...). Mais si Cinéma en Construction a voulu assez logiquement accompagner les renouveaux cinématographiques (en Argentine au début des années 2000, au Chili et en Colombie ensuite), il n'en est pas moins vrai que le soutien professionnel ne s'arrête pas aux poids lourds du continent, avec la sélection régulière de films « isolés » : c'est le cas du premier long-métrage du guatémaltèque Julio Hernández Cordón, Gasolina, découvert par la plateforme en 2007, et dont son dernier et septième film, Cómprame un revólver, a été sélectionné à la Quinzaine des Réalisateurs en 2018.

Schéma 1 : Pourcentage de films visionnés à Cinéma en Construction entre 2011 et 2017

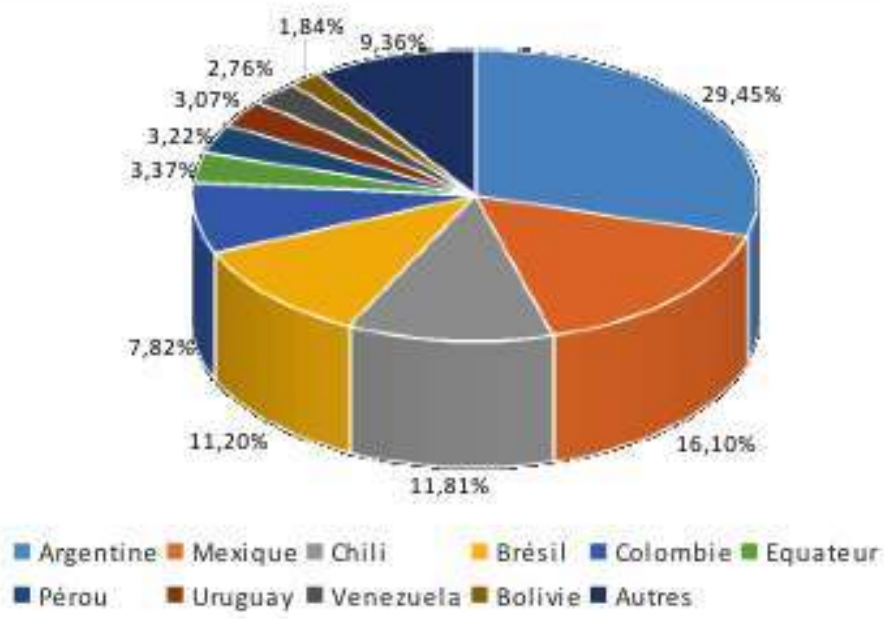

Source : Arcalt

La plateforme bénéficie actuellement du concours des collectivités territoriales et du Centre National du Cinéma et de l'image animée, CNC, qui lui attribue le label de « festival d'intérêt général ». Fidèle aux principes de l'exception culturelle française, ce soutien manifeste la convergence des intérêts publics et privés - ceux des professionnels d'un marché qui se reconfigure au gré des rencontres internationales. Le soutien public se traduit en termes de partenariat au niveau de la production cinématographique puisque le festival participe en retour à la dynamisation de la filière. De fait, l'action de Cinélatino se décline en données observables en mesure de rendre compte d'un interventionnisme qui touche désormais toute la chaîne de la production cinématographique.

Tableau 1 : Actions du festival Cinélatino au sein du marché

\begin{tabular}{|l|l|}
\hline Secteurs concernés & Actions du festival Cinélatino au sein de la filière \\
\hline Distribution & Prix Coup de cœur \\
\hline Exploitation & Projet Cinélatino en Région \\
\hline Exploitation & Promotion et communication sorties des films \\
\hline Post-production & Cinéma en Construction \\
\hline Développement & Cinéma en Développement \\
\hline
\end{tabular}


Production (FR 3, Région, Cinélatino) Appel à projets de documentaire

11 Du côté des réalisateurs, ce n'est plus tant l'achèvement du film qui est recherché que la rencontre avec des professionnels susceptibles de contribuer au développement d'un projet. En effet, depuis 2006, le dispositif Cinéma en Développement, réalisé en collaboration avec les Ateliers du Cinéma Européen et la Cinéfondation ${ }^{11}$, est venu compléter la plateforme dédiée à l'accompagnement des projets de films. Son objectif est de mettre en relation des professionnels désireux de découvrir des projets avec des réalisateurs ou des producteurs qui souhaitent nouer ou renforcer leur réseau de contacts professionnels. Lors de l'édition 2018, 22 projets ont bénéficié des rendez-vous organisés dans ce cadre.

D'autres actions mises en place chaque année par la plateforme professionnelle de Cinélatino répondent à cet objectif: le module Connexion Hub dont l'objectif est de donner des clefs pour appréhender le fonctionnement de l'industrie par le biais de rendez-vous entre des porteurs de projets latino-américains et des professionnels du secteur; la plateforme Occitania, en partenariat avec FR 3 Occitanie et la Région Occitanie, dont l'objectif est de soutenir la production documentaire régionale avec un appel à projets en partenariat avec l'Association des Producteurs Indépendants de la Région Occitanie/Pyrénées-Méditerranée (APIFA) autour du «documentaire en salle »; enfin, les séances de " prévisionnement pour les exploitants ", visant l'accompagnement des exploitants à la programmation des films latino-américains à travers la projection de 12 films sélectionnés par le Festival et sortis en salle la même année. L'intégration des projets de la région vise ainsi à renforcer les liens avec les professionnels du territoire.

En outre, en tant qu'acteur vital du réseau de production et de circulation commerciale des films latino-américains en France, Cinéma en Construction a pu bénéficier entre 2009 et $2011 \mathrm{du}$ soutien du programme européen Média Mundus. Pendant trois ans, la plateforme s'est déployée à une échelle plus ambitieuse à travers Cine Sin Fronteras, qui recouvre trois dispositifs: Cinéma en Construction, l'Atelier Cinemalab et le network EDEN (un réseau de distributeurs européens focalisé sur les films d'Amérique latine). La fin de cette subvention révèle les mutations des politiques européennes en matière de financement des industries culturelles.

\section{Circulation et notoriété internationales des films versus désaveu national}

Le dispositif Cinéma en Construction a des retombées sur la circulation, distribution et exploitation des films. En effet, la plupart des projets retenus sont menés à bien et sont alors en mesure d'accéder à une circulation qui va parfois jusqu'à l'exploitation commerciale (69 films ont été distribués en France depuis la création de la plateforme). Reconnaissons cependant, comme le rappelle Cédric Lépine (2017), qu'il s'agit là de « chiffres modestes d'exploitation en salles »: 25 films parmi les 711 longs-métrages et programmes courts sortis en salles en 2017 pour un total de 676538 entrées ${ }^{12}$.

15 L'internationalisation de la production se fonde ainsi sur un réseau large d'acteurs et de dispositifs de soutien permettant qu'un même projet puisse bénéficier du soutien de plusieurs fonds. L'appareil financier qui en résulte s'avère alors d'une grande complexité. 
Grâce à l'accord de coproduction entre la société de production colombienne Contravía et la société mexicaine Film Tank, établi pendant le Festival de Guadalajara, La Sirga (2012) de William Vega obtient par exemple le soutien du Fonds Ibermedia et du Fonds Sud en France. Une fois monté, il obtient le Prix Cinéma en Construction de l'Industrie. Prenons aussi le cas du deuxième long-métrage de Marcela Said, Los Perros. Produit par la société de production Cinéma Defacto, il a bénéficié de plusieurs sélections et du soutien des fonds pour le développement, la production et la postproduction: Sundance Lab, Procirep-Angoa et Aide aux cinémas du monde en France, MEDIA Slate funding, Fond audiovisuel chilien, World cinéma fund, Ibermedia, ICA (Aide à la coproduction francoportugaise) et NRW Filmstiftung allemand. Et il est également sélectionné à Cinéma en Construction.

En outre, la sélection d'un film à Cinéma en Construction lui assure souvent une belle carrière internationale une fois le film achevé. Celui qui obtient le Prix Industrie à San Sebastián fait partie d'une de sélections de ce festival : soit la sélection en compétition Zabaltegi du premier ou du deuxième film, soit la Sélection Officielle pour des cinéastes confirmés, soit alors la section parallèle Horizontes Latinos lorsqu'il n'a pas été sélectionné auparavant dans d'autres festivals. Il peut également participer à la section en Compétition ou aux sections parallèles du Festival Cinélatino. Le circuit festivalier ne se réduit pas pour autant à ces deux festivals. Les rendez-vous annuels dans les plateformes professionnelles de Rotterdam, de la Berlinale, de Locarno, de Ventana Sur, sont désormais incontournables pour la programmation des sections Compétition et Panorama de ces festivals. Un vivier de films constitué dans les sections professionnelles des festivals alimente de facto les sélections officielles. À son tour, la section Cinéma en Construction de Toulouse représente pour d'autres festivals internationaux une référence en matière de films latino-américains. Pour donner un exemple du tremplin que Cinéma en Construction représente pour la carrière internationale d'un film, sur les 189 films soutenus depuis la création, 22 ont été ensuite sélectionnés à Cannes et 19 au Festival de Berlin $^{13}$.

Mais si les relations étroites tissées à l'international contribuent à l'essor des cinématographiques nationales, il faut reconnaître que la réalité internationale est parfois bien éloignée des réalités du continent. Alors que les coproductions se multiplient et que plusieurs films circulent et sont primés dans des festivals internationaux, la place de ces films dans les marchés nationaux ou domestiques n'est guère reluisante, selon les mots d'Eva Morsh Kihn ${ }^{14}$. Des films défendus par les professionnels et les festivals internationaux ne connaissent pas toujours un succès similaire à l'intérieur des frontières nationales, comme le montre l'accueil des films colombiens La Sirga (21 538 spectateurs $^{15}$ pour 5872 en France ${ }^{16}$ ) et La Playa D.C. (16 015 spectateurs pour 6584 en France), entre autres. La situation inverse est également vraie. Les films les plus populaires qui ont fait le box-office national en Argentine, au Brésil, au Mexique ou en Colombie, n'ont pas leur place dans les plateformes professionnelles des festivals européens. Du point de vue des producteurs nationaux, ces plateformes et plus largement les dynamiques de production et de circulation cinématographiques internationales représentent une stratégie éprouvée pour réaliser et faire exister leurs films, car ceux-ci ne parviennent pas dans la plupart des pays, à l'exception peut-être de l'Argentine, à développer des marchés nationaux. D'après le producteur et réalisateur Oscar Ruíz Navia, en l'absence d'un marché favorable au cinéma d'auteur à l'intérieur de leur propre pays, il ne reste plus aux jeunes cinéastes colombiens qu'à s'ouvrir au public « des différentes parties du monde ${ }^{17}$ ». 
Reste la question qui hante chercheurs et critiques, celle de la normalisation des films du Sud pour les pays du Nord ${ }^{18}$.

\section{Un « mode culturel de production »}

18 La multiplication de ces plateformes et donc des films en circulation comme la configuration des réseaux dans plusieurs régions du monde, assurent au film latinoaméricain d'auteur un mode de production et de circulation transnational viable : argent public, soutien des acteurs de l'industrie et de la technique internationaux, sociétés de production locales et, bien entendu, plateformes professionnelles régionales dans plusieurs pays du continent latino-américain et international.

Si certains professionnels utilisent le terme d'industrie pour désigner le retentissement de ce marché et la professionnalisation des pratiques, la taille et la fragilité des vendeurs et des sociétés de production et de distribution impliquées relèvent davantage de l'artisanat que d'un modèle industriel. Ce mode de production se fonde sur deux valeurs : premièrement, celle de l'indépendance, dans son acception française, qui « incarne une alternative à l'universel marchand et dérégulé représenté par les États-Unis ${ }^{19}$ ». Indépendance donc vis-à-vis du modèle de production et des formes cinématographiques mainstreams du cinéma nord-américain, d'une part, et des cinématographies domestiques hégémoniques, d'autre part.

Le "cinéma d'auteur" constitue la deuxième valeur qui côtoie et conforte celle d'indépendance dans le discours des acteurs de ce marché. La participation des professionnels à ce marché témoigne d'une "communauté de pensée artistique et culturelle indépendante ${ }^{20} »$, engagée dans le processus de création et la défense d'un " cinéma d'auteur ». Lieu de partage de valeurs lesté d'enjeux économiques, Cinéma en Construction place la question des rapports cinématographiques dans un cadre en réalité plus complexe que celui des rapports de domination coloniaux. L'expression "cinéma d'auteur latino-américain » dépasse en l'occurrence celle de "cinémas du Sud » et son corollaire, le clivage géo-esthétique Nord-Sud, et amène à penser autrement cette relation.

21 L'aide à l'existence des cinémas d'Amérique latine, principal mobile de Cinéma en Construction, se double désormais du recours à la rhétorique en vogue de la diversité culturelle qui tend à redéfinir les contours de l'engagement politique qui a marqué l'histoire du festival de Toulouse. La présentation de la $33^{\text {ème }}$ édition de la plateforme professionnelle en mars 2018 est à ce titre assez parlante :

22 En impliquant des professionnels capables de contribuer à la post-production et en encourageant les distributeurs et les diffuseurs à la diversification de l'offre, San Sebastián et Toulouse contribuent à développer et à consolider, avec pragmatisme et efficacité, le respect et la promotion de la diversité culturelle fondée sur un esprit de solidarité et de coopération ${ }^{21}$.

23 En tant qu'instrument de promotion de la différence, le concept de diversité culturelle s'avère un nouvel horizon des acteurs des circuits transnationaux du marché culturel ${ }^{22}$. D'un point de vue plus global, il semble s'inscrire dans une double stratégie: la commercialisation des produits culturels à l'heure de la mondialisation ${ }^{23}$ et la diplomatie marchande française ${ }^{24}$. 

la défense et de la promotion de la diversité culturelle, du cinéma indépendant et du cinéma d'auteur, un tournant manifeste : en tant qu'espaces de rencontre des acteurs du marché, elles affirment sans complexe que l'économie se trouve au cœur des échanges. Il s'agirait, pour reprendre l'expression de Thomas Elssaesser ${ }^{25}$, d'un «mode culturel de production » qui consiste à mettre en avant « un cinéma local » (contribuant peut-être ainsi à redéfinir le cinéma national) à des fins d'exportation, c'est-à-dire pour le marché mondial. Alors qu'au début des années 2000, cette situation soulevait des interrogations inédites sur l'écart entre un cinéma «international " et un cinéma "local ${ }^{26}$ ", les professionnels du cinéma d'auteur assument aujourd'hui la dialectique local/ transnational, et le soupçon d'une tentation mimétique par rapport à un «modèle du centre » se résout souvent à travers l'affirmation des histoires locales.

\section{Conclusion}

Les plateformes professionnelles festivalières, apparues dans le courant des années 2000 d'abord en Europe et ensuite en Amérique latine, agissent comme des instances de médiation privilégiées des films et des cinéastes latino-américains à l'intérieur du marché cinématographique français et européen dit «indépendant", en même temps qu'elles révèlent les nouveaux enjeux économiques et politiques à l'œuvre dans les échanges cinématographiques France/Amérique latine.

Depuis son origine en 1989, le Festival Cinélatino Rencontres de Toulouse, anciennement appelé Rencontres des Cinémas d'Amérique latine, s'est attaché à la promotion des cinémas de ces pays. La création de Cinéma en Construction après quatorze éditions lui a permis de réaffirmer ses objectifs tout en les affinant: il s'agissait en l'occurrence de faciliter les échanges entre professionnels de deux côtés de l'Atlantique et de promouvoir l'intérêt des professionnels du cinéma français et plus largement européen pour la production, la circulation et la distribution commerciale des films du continent latinoaméricain.

27 Avec d'autres espaces de rencontre, de négociation et de circulation des projets et des films, ils ont façonné au cours des deux dernières décennies un réseau de coopération, au sens que lui attribue Howard Becker ${ }^{27}$ pour définir les mondes de l'art : professionnels, institutions, festivals et plateformes professionnelles, écoles et lieux de formation participent tous de ce qui constitue le monde du cinéma d'auteur contemporain latinoaméricain.

Parce qu'elles promeuvent des dynamiques professionnelles inédites, qu'elles imposent le calendrier de la production, qu'elles agissent comme des dispositifs de découverte de jeunes auteurs et de rencontre où se définit, se négocie et s'évalue la cote des films et des cinéastes, mais aussi parce qu'elles donnent un élan à la coproduction entre les pays européens et latino-américains, ces plateformes rendent compte d'un savoir-faire partagé en matière d'industrie cinématographique.

L'approche de Cinéma en Construction et plus globalement des dynamiques du marché du film latino-américain mettent en lumière la dialectique du secteur, partagée entre la volonté d'élargir la production et la circulation des films à l'échelle européenne et le souhait d'affirmer la dimension authentique de ces cinémas. 


\section{BIBLIOGRAPHIE}

Olivier Alexandre, Noël Sophie et Pinto Aurélie (sous la dir.), « Introduction », dans Culture et indépendance. Les enjeux de l'indépendance dans les industries culturelles, Bruxelles, P.I.E. Peter Lang, 2017, p. $9-16$.

Nicolas Azalbert, 2004. «Le nouvel enjeu argentin », dossier « Amérique latine : dans le sillage de l'Argentine, le réveil d'un continent », Cahiers du Cinéma, n 590, 2004, p. 36 - 37.

Howard Becker, Les Mondes de l'art, Champs arts, Paris, 2006.

Minerva Campos, «La América Latina de “Cine en Construcción” Implicaciones del apoyo económico de los festivales internacionales ", Archivos de la filmoteca, n 71, 2013, p. 13 - 26.

Pierre Delcambre, "Penser des pratiques culturelles en se saisissant du concept de médiation », Études de communication, 21, 1998, p. 137 - 144.

Thomas Elsaesser, « "ImpersoNations” : cinéma national, imaginaires historiques et nouveau cinéma européen », Mise au point [En ligne], 5 | 2013, mis en ligne le 01 avril 2013, consulté le 21 février, 2018. URL : http://journals.openedition.org/map/1315 ; DOI : 10,400 0/map.13

Romain Lecler, « Nouvelles vagues. Le marché-festival de Cannes ou la fabrique française d'un universal cinématographique ", Actes de la recherche en sciences sociales, n² 206 - 207, mars 2015, p. $15-33$.

Romain Lecler, "Une diversité sur mesure. Les conditions d'existence d'un cinéma du "Sud" ", Sociologie, 2017/2 (Vol. 8), p. 139-160.

Cédric Lepine, « Distribution et exploitation du cinéma latino en France en 2017 », Médiapart, publié le 14 mars 2018.

André Mondoux, Jean-Guy Lacroix, « Fausse et vraie diversité culturelle dans le systèmemonde », dans Yves Théorêt (dir. publ.) David contre Goliath : La Convention sur la protection et la promotion de la diversité des expressions culturelles de l'UNESCO, Montréal, Éditions Hurtubise, 2009, p. $76-98$.

Michel Reilhac, «L'International du cinéma indépendant », dans Frodon Jean-Michel (dir.), Au Sud du cinéma. Films d'Afrique, d'Asie et d'Amérique latine, Cahiers du cinéma, Arte Editions, Paris, 2004, p. $17-21$.

Amanda Rueda, « Festival de cinéma. Médiations et construction de territoires imaginaires », Culture \& musées, $\mathrm{n}^{\circ} 14,2009$, p. 147 - 171.

Amanda Rueda, «L'Amérique latine en France. Festivals de cinéma et territoires imaginaires, Toulouse, Presses universitaires du Midi, 2018, p. 269 - 316.

\section{NOTES}

1. L'Aide aux cinémas du monde en France, ancien Fonds du Sud (1984 - 2011), ou bien encore Ibermedia, programme de soutien à la coproduction de films réunissant dix-neuf pays d'Amérique du Sud, l'Espagne et le Portugal, né en 1998. 
2. Amanda Rueda, « Festival de cinéma. Médiations et construction de territoires imaginaires », Culture \& musées, n 14, 2009, p. 149 - 167.

3. Michel Reilhac, «L'International du cinéma indépendant », dans Frodon Jean-Michel (dir.), Au Sud du cinéma. Films d'Afrique, d'Asie et d'Amérique latine, Cahiers du cinéma/ Arte Editions, Paris, 2004, p. 17.

4. Amanda Rueda, op.cit. p. 149.

5. Pierre Delcambre, «Penser des pratiques culturelles en se saisissant du concept de médiation », Etudes de communication, 21, 1998, p. 143.

6. Amanda Rueda, 2009, op.cit. p. 158.

7. Romain Lecler, « Nouvelles vagues. Le marché-festival de Cannes ou la fabrique française d'un universal cinématographique », Actes de la recherche en sciences sociales, $\mathrm{n}^{\circ}$ 206 - 207, mars 2015, p. 15 - 33.

8. Campo, Minerva, « La América Latina de "Cine en Construcción”. Implicaciones del apoyo económico de los festivales internacionales ", Archivos de la filmoteca, n 71, 2013, p. 15.

9. Entretien avec Esther Saint-Dizier, membre fondatrice du Festival Cinélatino Rencontres de Toulouse et du comité de sélection de Cinéma en Construction, le 7 décembre 2017.

10. Les partenaires du Prix Cinéma en Construction à Toulouse sont actuellement le CNC (qui attribue une bourse qui sert à la prise en charge des travaux de post-production en France), le CCAS (qui offre une résidence de quatre semaines au lauréat pour qu'il puisse assurer la post-production ou la promotion du film en France), Eaux Vives Productions (coordination de la post-production du film lauréat), Commune Image et FireFly Cinéma (qui offrent l'étalonnage), Mactari Studio (la post-production son), Titra Films (le soustitrage).

11. La Résidence Cinéfondation du Festival de Cannes, créée en 2000, accueille chaque année au cours de deux sessions une douzaine de jeunes réalisateurs originaires des pays dits du Sud ou de l'Est, qui travaillent sur leur projet de premier ou deuxième longmétrage de fiction. http://www.cinefondation.com/fr

12. Données statistiques de fréquentation de CBO Box Office analysées par Cédric Lepine dans son blog sur les Cinémas d'Amérique latine, Médiapart, 14 mars 2018 : « Distribution et exploitation du cinéma latino en France en 2017 ».

13. Catalogue $29^{\text {es }}$ Cinélatino Rencontres de Toulouse, mars 2017, p. 89.

14. Entretien avec Eva Morsh Kihn, coordinatrice de la plateforme professionnelle du Festival Cinélatino Rencontres de Toulouse, le 16 janvier 2018.

15. http://www.mincultura.gov.co/areas/cinematografia/estadisticas-del-sector/ Documents/Hist\%C3\%B3rico\%20Pel\%C3\%ADculas\%20Colombianas\%20Estrenadas\% 201996-2016.pdf

16. Données obtenues grâce à l'aide précieuse de Laurent Coudurier de CBO Box Office.

17. Brochure commémorative des dix premières années de la société de production Contravía Films à Cali (Colombie).

18. Romain Lecler, « Une diversité sur mesure. Les conditions d'existence d'un cinéma du «Sud » », Sociologie, 2017/2 (Vol. 8), p. 139-160.

19. Olivier, Alexandre, Sophie Noël, et Aurélie Pinto (sous la dir.), « Introduction », dans Culture et indépendance. Les enjeux de l'indépendance dans les industries culturelles, Bruxelles, P.I.E. Peter Lang, 2017, p. 15. 
20. Jean Roatta, cité par Romain Lecler, « Une diversité sur mesure. Les conditions d'existence d'un cinéma du « Sud » », op. cit. p. 148.

21. http://www.cinelatino.fr/contenu/inscription-cinema-en-construction-33

22. Amanda Rueda, «Le renouvellement des cinématographies nationales ", L'Amérique latine en France. Festivals de cinéma et territoires imaginaires, Toulouse, Presses universitaires du Midi, 2018.

23. André Mondoux, Jean-Guy Lacroix, «Fausse et vraie diversité culturelle dans le système-monde ", dans Yves Théorêt (dir. publ.) David contre Goliath : La Convention sur la protection et la promotion de la diversité des expressions culturelles de l'UNESCO, Montréal, Éditions Hurtubise, 2009, pp. 76 - 98.

24. Romain Lecler, 2017 », op. cité. pp.139-160.

25. Thomas Elsaesser, « ImpersoNations » : cinéma national, imaginaires historiques et nouveau cinéma européen ", Mise au point [En ligne], 5 | 2013, mis en ligne le 01 avril 2013, consulté le 21 février, 2018. URL : http://journals.openedition.org/map/1315 ; DOI : 10.4000/map.13

26. Nicolas Azalbert, « Le nouvel enjeu argentin », Dossier « Amérique latine : dans le sillage de l'Argentine, le réveil d'un continent », Cahiers du Cinéma, n 590, 2004, p. 37. 27. Howard Becker, Les mondes de l'art, Champs arts, Paris, 2006.

\section{RÉSUMÉS}

Les trois dernières décennies ont été marquées par l'évolution des festivals de cinéma, notamment de ceux qui s'intéressent aux cinémas d'Amérique latine, vers un compagnonnage qui va bien au-delà de la programmation et de la diffusion des films à travers la création de plateformes professionnelles où se rencontrent les différents acteurs du champ (cinéastes, producteurs, distributeurs, vendeurs, programmateurs d'autres festivals). Devenues des rendezvous obligés pour cs professionnels, notamment français, intéressés par ces cinémas, ces plateformes entraînent de mutations dans les pratiques de coopération et de coproduction cinématographique avec les pays d'Amérique latine. Il s'agit dans cet article d'explorer la plateforme professionnelle Cinéma en Construction, des festivals Cinélatino Rencontres de Toulouse et San Sebastian, en tant qu'espace de médiation au sein du marché du film d'auteur latino-américain. Nous tenterons d'éclairer les enjeux de ce rendez-vous dans la construction de ce que d'aucuns n'hésitent pas à qualifier d'« industrie internationale du cinéma d'auteur ».

The last three decades have been marked by the evolution of film festivals, especially those dedicated to Latin American cinemas, towards a mentoring network that goes beyond the exhibition and distribution of films. It follows the creation of professional platforms where the different actors of the sector meet (filmmakers, producers, distributors, sellers, other festivals' programmers). These platforms have become unmissable events for the professionals (especially the French professionals) interested in these cinemas. They have contributed to deeply change the practices of film cooperation and film co-production with Latin American countries. This paper explores the professional platform "Cinéma en Construction" (Films in progress), a joint initiative of Cinélatino, Rencontres de Toulouse and San Sebastian Festival, as a space of 
mediation within the Latin-American auteur film market. We will examine this event in the light of what some professionals don't hesitate to call an "international industry of auteur films".

\section{AUTEUR}

\section{AMANDA RUEDA}

Maîtresse de conférences en Sciences de l'information et de la communication à l'Université Toulouse 2 Jean Jaurès, attachée au laboratoire LERASS. Ses travaux portent sur les écritures audiovisuelles et sur les processus de création et de production des cinématographies d'Amérique latine. Auteure l'ouvrage L'Amérique latine et France. Festivals de cinémas et territoires imaginaires (PUM, 2018). 